\title{
Spin, quadrupole moment, and deformation of the magnetic-rotational band head in ${ }^{193} \mathrm{~Pb}$
}

\author{
D. L. Balabanski, ${ }^{1,2, *}$ M. Ionescu-Bujor, ${ }^{3}$ A. Iordachescu, ${ }^{3}$ D. Bazzacco, ${ }^{4}$ F. Brandolini, ${ }^{4}$ D. Bucurescu, ${ }^{3}$ S. Chmel,${ }^{5, \dagger}$ \\ M. Danchev, ${ }^{6}$ M. De Poli, ${ }^{7}$ G. Georgiev,${ }^{8}$ H. Haas, ${ }^{9,10}$ H. Hübel,${ }^{5}$ N. Marginean,${ }^{3,4,7}$ R. Menegazzo, ${ }^{4}$ G. Neyens, ${ }^{1}$ \\ P. Pavan, ${ }^{4}$ C. Rossi Alvarez, ${ }^{4}$ C. A. Ur, ${ }^{3,4}$ K. Vyvey, ${ }^{1}$ and S. Frauendorf ${ }^{11}$ \\ ${ }^{1}$ Instituut voor Kern-en Stralingsfysica, K.U. Leuven, B-3001 Leuven, Belgium \\ ${ }^{2}$ INRNE, Bulgarian Academy of Sciences, BG-1784 Sofia, Bulgaria \\ ${ }^{3}$ National Institute for Physics and Nuclear Engineering, R-76900 Bucharest, Romania \\ ${ }^{4}$ Dipartimento di Fisica dell' Università and INFN, Sezione di Padova, I-35131 Padova, Italy \\ ${ }^{5}$ Helmholtz-Institut für Strahlen- und Kernphysik, Universität Bonn, D-53115 Bonn, Germany \\ ${ }^{6}$ Faculty of Physics, St. Kliment Ohridski University of Sofia, BG-1164 Sofia, Bulgaria \\ ${ }^{7}$ INFN, Laboratori Nazionali di Legnaro, I-35020 Legnaro, Italy \\ ${ }^{8}$ Centre de Spectrométrie Nucléaire et de Spectrométrie de Masse, F-91405 Orsay-Campus, France \\ ${ }^{9}$ Hahn-Meitner Institute, Bereich Festkoperphysik, D-14109 Berlin, Germany \\ ${ }^{10}$ EP Division, CERN, CH-1211 Geneva, Switzerland \\ ${ }^{11}$ Department of Physics, University of Notre Dame, Notre Dame, Indiana 46556, USA
}

(Received 10 August 2009; revised manuscript received 3 October 2010; published 18 January 2011)

\begin{abstract}
The spectroscopic quadrupole moment of the $T_{1 / 2}=9.4(5) \mathrm{ns}$ isomer in ${ }^{193} \mathrm{~Pb}$ at an excitation energy $E_{\mathrm{ex}}=$ $(2585+x) \mathrm{keV}$ is measured by the time-differential perturbed angular distribution method as $\left|Q_{s}\right|=2.6(3) e \mathrm{~b}$. Spin and parity $I^{\pi}=27 / 2^{-}$are assigned to it based on angular distribution measurements. This state is the band head of a magnetic-rotational band, described by the coupling of a neutron hole in the $1 i_{13 / 2}$ subshell with the $\left(3 s_{1 / 2}^{-2} 1 h_{9 / 2} 1 i_{13 / 2}\right)_{11^{-}}$proton excitation. The pairing-plus-quadrupole tilted-axis cranking calculations reproduce the measured quadrupole moment with a moderate oblate deformation $\epsilon_{2}=-0.11$, similar to that of the $11^{-}$proton intruder states, which occur in the even-even $\mathrm{Pb}$ nuclei in the region. This is the first direct measurement of a quadrupole moment and thus of the deformation of a magnetic-rotational band head.
\end{abstract}

DOI: 10.1103/PhysRevC.83.014304 PACS number(s): 21.10.Ky, 21.10.Tg, 21.60.Ev, 27.80.+w

\section{INTRODUCTION}

The occurrence of regular rotational-like sequences of strongly enhanced magnetic dipole $(M 1)$ transitions, in the vicinity of closed shells, is a very interesting nuclear structure feature which was subject of much experimental and theoretical work (for review, see Refs. [1-3]). In these bands, the $M 1(\Delta I=1)$ transitions are very strong, whereas the $E 2(\Delta I=2)$ crossover transitions are weak or not even observed. The ratios of reduced transition probabilities, $B(M 1) / B(E 2)$, derived from the $\gamma$-ray intensity ratios are unusually large, around $25 \mu_{N}^{2} /(e \mathrm{~b})^{2}$. Lifetime measurements showed that the $M 1$ transitions are strongly enhanced. The $B(M 1)$ values are large (of the order of few $\mu_{N}^{2}$ ) and decrease with spin within the bands [2,3], while the $B(E 2)$ values are small. This type of band, the "magnetic rotational band" [4,5], arises from a spontaneous symmetry breaking by anisotropic currents of a few high- $j$ configurations (particles and holes). The perpendicular orientation of their angular momenta with respect to each other gives the lowest energy, which corresponds to the band head. Angular momentum toward higher energies along dipole bands is generated by the step-by-step alignment of the particle and hole spins in the direction of the total angular momentum [6], which results in a decrease of the $M 1$ strength within the bands. Since this

\footnotetext{
*Corresponding author: balabanski@inrne.bas.bg.
}

†Present address: Fraunhofer INT, D-53879 Euskirchen, Germany. resembles the closing of the blades of a shears, the $M 1$ bands have also been called "shears bands" [7].

Two theoretical approaches were proposed for the description of the observed experimental features: the tilted-axis cranking (TAC) model [4-6] and an empirical approach that assumes a particular effective interaction between high-spin particles and holes [2,8]. The TAC model is a mean-field approach that considers cases for which the angular momentum of a nucleus does not coincide with one of the principle axes. In the calculations, the parameters of the Hamiltonian are adjusted to reproduce the experimental deformations of a certain state. The empirical model assumes an effective quadrupole moment, taking into account the contributions from the protons and the neutrons by introducing proton and neutron effective charges, $e_{\pi}$ and $e_{\nu}$, respectively.

Magnetic-rotational bands were discovered in several mass regions, but the richest information exists for the $\mathrm{Pb}$ isotopes [1-3], where such bands were observed for the first time in ${ }^{199} \mathrm{~Pb}$ [9]. More than 50 bands have been reported in neutrondeficient $\mathrm{Pb}$ nuclei with mass numbers $191 \leqslant A \leqslant 202$. These $M 1$ bands are built on two-proton high- $j$ particle-hole excitations across the $Z=82$ shell gap coupled to neutron holes in the $1 i_{13 / 2}$ orbital and possible contribution of low- $j$ neutrons. The perpendicular orientation of the proton and neutron spins in these bands was confirmed by a $g$-factor measurement [10]. A further important aspect for understanding the $M 1$ bands concerns the underlying deformation. Small E2 transition probabilities, pointing to a small deformation, are deduced from the measured branching ratios. However, a precise 
determination of transition quadrupole moments is difficult due to the large uncertainty in the intensity of the weak $E 2$ crossover transitions. Model-independent information on the size of the deformation can be provided by measurements of the spectroscopic quadrupole moments.

The $v\left(1 i_{13 / 2}^{-1}\right) \otimes \pi\left(3 s_{1 / 2}^{-2} 1 h_{9 / 2} 1 i_{13 / 2}\right)_{11^{-}}$configuration and spin and parity $I^{\pi}=29 / 2^{-}$were assigned to the $T_{1 / 2}=$ $9.4(5) \mathrm{ns}$ band head of the yrast $M 1$ band in ${ }^{193} \mathrm{~Pb}$ [11-13]. The configuration was confirmed by a $g$-factor measurement [10]. The spin assignment, however, differs from the systematic of similar bands in the $\mathrm{Pb}$ nuclei $[14,15]$. Here we report the spectroscopic quadrupole moment of this state, the first such measurement for a magnetic-rotational band head. In addition, based on angular distribution (ADO) data, spin and parity $I^{\pi}=27 / 2^{-}$were assigned to it. Preliminary results of this work were presented at conferences $[16,17]$.

\section{EXPERIMENTAL PROCEDURE AND RESULTS}

The experiment was carried out at the XTU-Tandem of the INFN Legnaro National Laboratory. Excited states in ${ }^{193} \mathrm{~Pb}$ were populated and spin-aligned in the ${ }^{170} \operatorname{Er}\left({ }^{28} \mathrm{Si}, 5 n\right)$ reaction at $143 \mathrm{MeV}$. The ${ }^{28} \mathrm{Si}$ beam was pulsed with a pulse width of $1.5 \mathrm{~ns}$, a repetition period of $800 \mathrm{~ns}$, and a suppression of the continuous beam in between the beam bursts of $\approx 10^{4}$. The excited ${ }^{193} \mathrm{~Pb}$ nuclei recoiled out of the $0.5 \mathrm{mg} / \mathrm{cm}^{2}{ }^{170} \mathrm{Er}$ foil into the solid $0.2-\mathrm{mm} \mathrm{Hg}$ layer mounted on a $\mathrm{Cu}$ finger cooled down to a temperature of $T=170.0(1) \mathrm{K}$.

The quadrupole interaction (QI) was investigated in the electric field gradient (EFG) of solid polycrystalline $\mathrm{Hg}$ by applying the time-differential perturbed angular distribution (TDPAD) method. The reason for choosing the $\mathrm{Hg}$ host is that the EFG, $V_{z z}$, provided by its lattice at the substitutionally positioned $\mathrm{Pb}$ nuclei, is very high [18]; for example, for substitutionally positioned $\mathrm{Pb}$ nuclei in solid $\mathrm{Hg}, V_{z z}=17.4(9) \times 10^{21} \mathrm{~V} / \mathrm{m}^{2}$ at $T=170 \mathrm{~K}$ [19], which results in a strong interaction observable on the very short time scale given by the isomer lifetime.

The $\gamma$ rays were detected by planar HPGe detectors placed at angles of $0^{\circ}$ and $90^{\circ}$ with respect to the beam direction. Such a detector arrangement allows both TDPAD and angular distribution measurements. A time resolution between 8.5 and $6.5 \mathrm{~ns}$ was obtained for $\gamma$ rays in the range of 200 to $500 \mathrm{keV}$, respectively. For the analysis of the list-mode data, energy-time matrices were sorted for each detector. Time-gated energy spectra were created for the analysis of the prompt and delayed transitions. The prompt and a delayed $\gamma$-ray energy spectrum in the 15-30-ns interval after the beam burst are displayed in Fig. 1. While the prompt spectrum is dominated by the $\gamma$ rays from Coulomb excitation of the ${ }^{170} \mathrm{Er}$ target and the $\mathrm{Hg}$ backing, the spectrum registered in between the beam pulses clearly illustrates delayed $\gamma$ rays deexciting the short-lived isomers in ${ }^{193} \mathrm{~Pb}$, which are shown in Fig. 2.

In addition to the previously reported 9.4-, 180-, and 20.5-ns isomers [13,20], another isomer was established, the $27 / 2^{-}(2322+x) \mathrm{keV}$ level in ${ }^{193} \mathrm{~Pb}$, as shown in Fig. 2. The $\gamma$ rays, which deexcite it, are present in the delayed spectrum

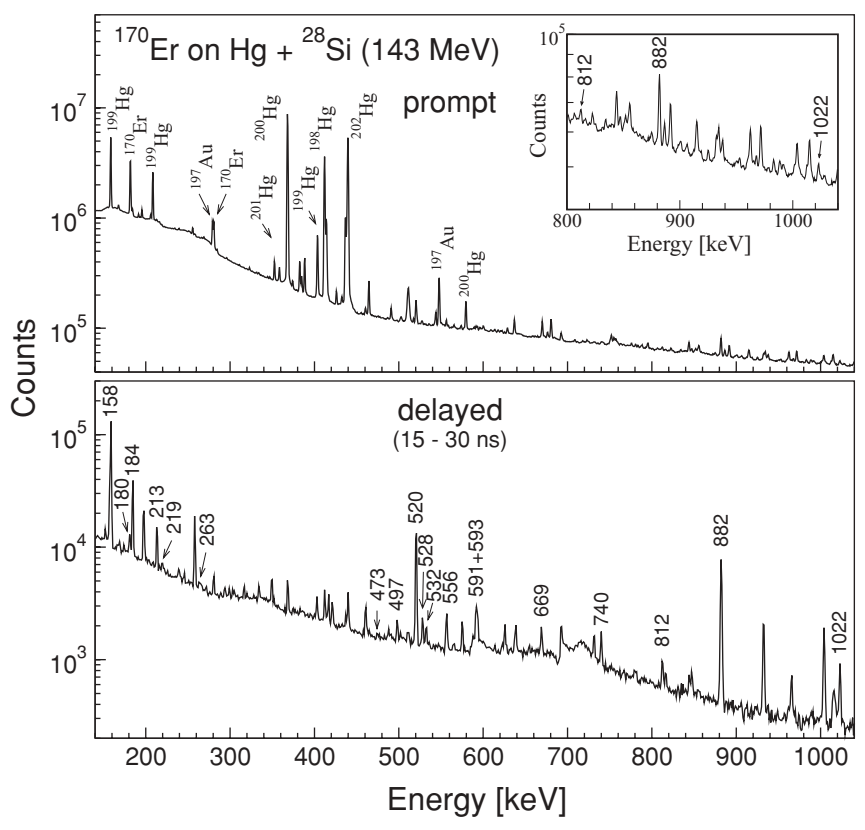

FIG. 1. Prompt and delayed $\gamma$-ray energy spectra registered with a planar HPGe detector. In the prompt spectrum, the strong Coulex $\gamma$ lines are labeled by the included nuclei. The isomeric decay $\gamma$ lines in ${ }^{193} \mathrm{~Pb}$ are labeled by their energies in $\mathrm{keV}$ in the delayed spectrum. The background due to long-lived activities has been subtracted. The inset shows some of the $\gamma$ rays that have been used in the ADO analysis (see text).

of Fig. 1. Time spectra of the 180- and 556-keV $\gamma$ rays are displayed in Fig. 3, from which a half-life $T_{1 / 2}=5.3(6) \mathrm{ns}$ was deduced for this state. A half-life $T_{1 / 2}=9.4(5) \mathrm{ns}$ was derived for the $M 1$ band head (see Fig. 3), in perfect agreement with the value reported in Ref. [10].

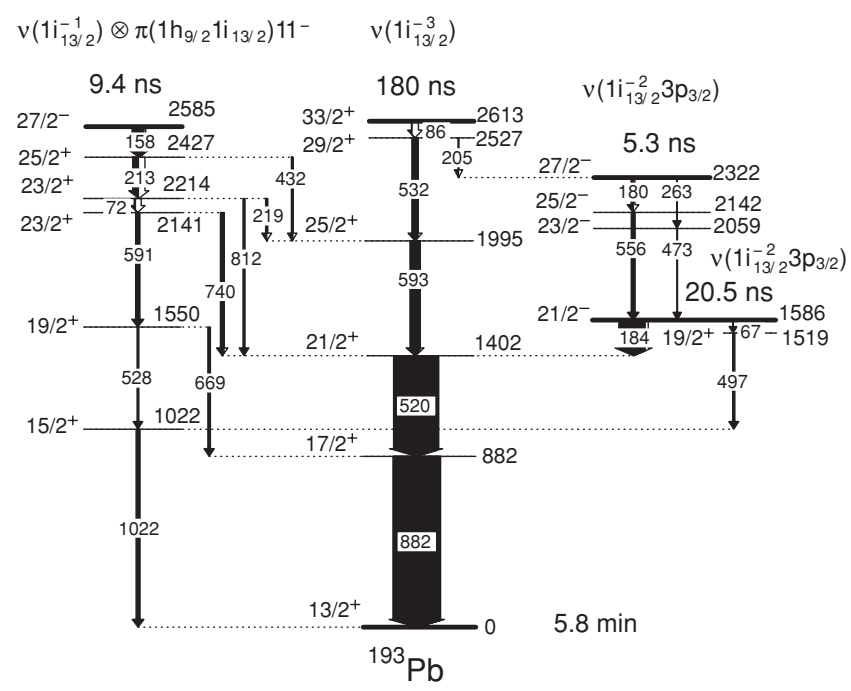

FIG. 2. The decay scheme of the short-lived isomers in ${ }^{193} \mathrm{~Pb}$ from Refs. [11-13,20] and present work. The $\gamma$ linewidth shows the isomeric decay components. The excitation energies are given in $\mathrm{keV}$ relative to the $13 / 2^{+}$long-lived isomer. The spins of the 2214-, 2427-, and $2585-\mathrm{keV}$ states were assigned in the present work. 


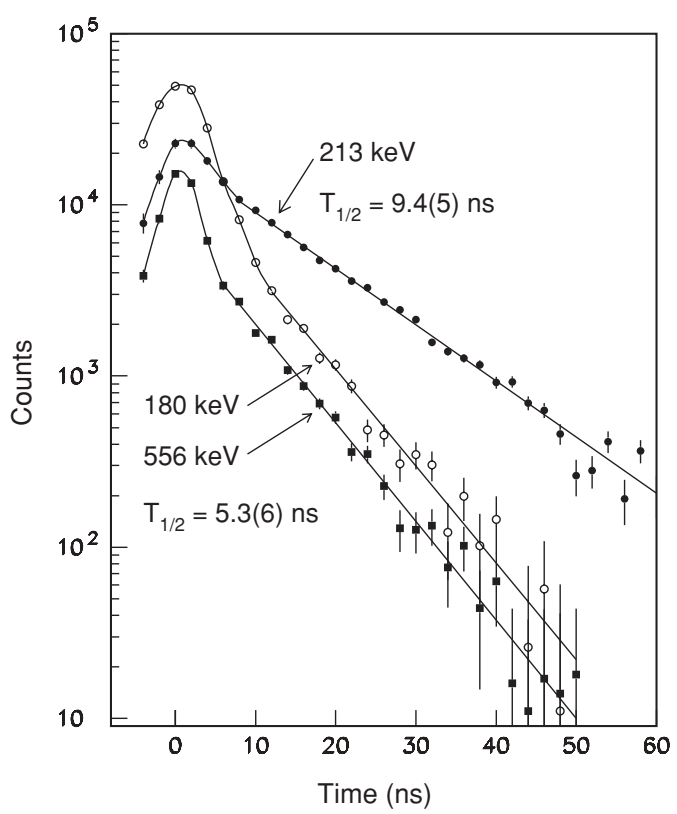

FIG. 3. Time spectra for the 213-, 180-, and 556-keV $\gamma$ lines showing the half-lives of the two $27 / 2^{-}$isomers at $(2585+x) \mathrm{keV}$ and $(2232+x) \mathrm{keV}$, respectively. The prompt and the longer-lived backgrounds are subtracted.

Previous multipolarity assignments for the $\gamma$ transitions involved in the isomeric decays in ${ }^{193} \mathrm{~Pb}$ were based on conversion coefficient [11], angular distribution [11,13], and directional correlation [12] measurements. Note that in some cases discrepancies were observed between the reported values in these studies. To check the assigned multipolarities, we analyzed the ratio of the $\gamma$ intensities, $R_{\mathrm{ADO}}=I_{\gamma}\left(0^{\circ}\right) / I_{\gamma}\left(90^{\circ}\right)$, in prompt spectra registered at the angles $0^{\circ}$ and $90^{\circ}$. This ratio takes values of $\approx 1.3$ for stretched $E 2$ transitions and $\approx 0.8$ for pure dipole stretched transitions. In the case of transitions of $M 1+E 2$ mixed character, $R_{\mathrm{ADO}}$ depends on the value and sign of the mixing coefficient $\delta$, being $R<0.8$ for $\delta<0$, and $R>0.8$ for $\delta>0$. The ADO analysis of the prompt spectra in the present experiment agreed with the adopted multipolarities for most of the transitions below the isomers [11-13]. Thus, $R_{\mathrm{ADO}}$ values of around 1.3 were obtained for the 882-, 520-, $528-$, and 556-keV transitions deexciting the lowest lying yrast states, in accordance with their E2 multipolarity. For the $1022-$ and $740-\mathrm{keV}$ transitions, the derived $R_{\mathrm{ADO}}$ values were $0.9(1)$ and $0.5(1)$, confirming their mixed $M 1+E 2$ character [11-13]. In the same time, for the $812-\mathrm{keV}$ transition, which was previously assigned as $E 2, R_{\mathrm{ADO}}=1.0(1)$ was deduced, indicating a mixed $M 1+E 2$ multipolarity. Note that in the present measurement, the good energy resolution of the used detectors ( $1.5 \mathrm{keV}$ for $800-\mathrm{keV}$ region) allowed better separation of the peaks in the rather complex spectra (see the inset of Fig. 1), which gives support to the analysis. Based on the present result for the $812-\mathrm{keV}$ transition, spin-parity $I^{\pi}=23 / 2^{+}, I^{\pi}=25 / 2^{+}$, and $I^{\pi}=27 / 2^{-}$are assigned to the 2214- and $2427-\mathrm{keV}$ states and the 9.4-ns isomer (see Fig. 2), $1 \hbar$ less compared to previous work [11-13]. With the new spin assignments, the states of the yrast $M 1$ band fit well into the energy systematics of such bands in the $\mathrm{Pb}$ nuclei $[14,15]$.

For the TDPAD analysis, energy-gated time spectra were used to construct the experimental ratios

$$
R_{\text {expt }}(t)=\frac{I\left(t, 0^{\circ}\right)-I\left(t, 90^{\circ}\right)}{I\left(t, 0^{\circ}\right)+I\left(t, 90^{\circ}\right)}
$$

which were least-squares fitted to the expression

$$
R_{\text {theor }}(t)=\frac{3}{4} A_{2} \sum_{n=1}^{\infty} S_{2 n} \cos \left(n \omega_{0} t\right)
$$

with the angular distribution coefficient $A_{2}$ and the quadrupole interaction frequency $\omega_{0}$ as free parameters. The QI frequency for a half-integer spin is $\omega_{0}=\frac{3 \pi v_{Q}}{I(2 I-1)}$, where $v_{Q}=e Q_{s} V_{z z} / h$ is the quadrupole coupling constant. The spin-dependent $S_{2 n}$ coefficients are calculated for an axially symmetric randomly oriented EFG [21].

In the present experiment, the $25 / 2^{+} \rightarrow 23 / 2^{+} 213-\mathrm{keV}$ transition was used in the analysis, because the $158.4-\mathrm{keV}$ transition that deexcites the 9.4-ns isomer is contaminated by the intense $158.4-\mathrm{keV}$ Coulex line in ${ }^{199} \mathrm{Hg}$ with $T_{1 / 2}=2.45(3)$ ns [22] (see Fig. 1). The 213-keV transition has a small prompt component, as seen in Fig. 3, and therefore the perturbed pattern could be followed very close to $t=0$, which makes it very convenient for TDPAD analysis. The experimental and theoretical $R(t)$ functions are displayed in the upper panel of Fig. 4. Owing to the high-spin value and the short lifetime of the isomer, it was possible to observe only the modulation pattern at the beginning of the quadrupole period $T_{0}=2 \pi / \omega_{0}$. A value $v_{Q}=1.11(9) \mathrm{GHz}$ was derived for the quadrupole coupling constant, resulting in
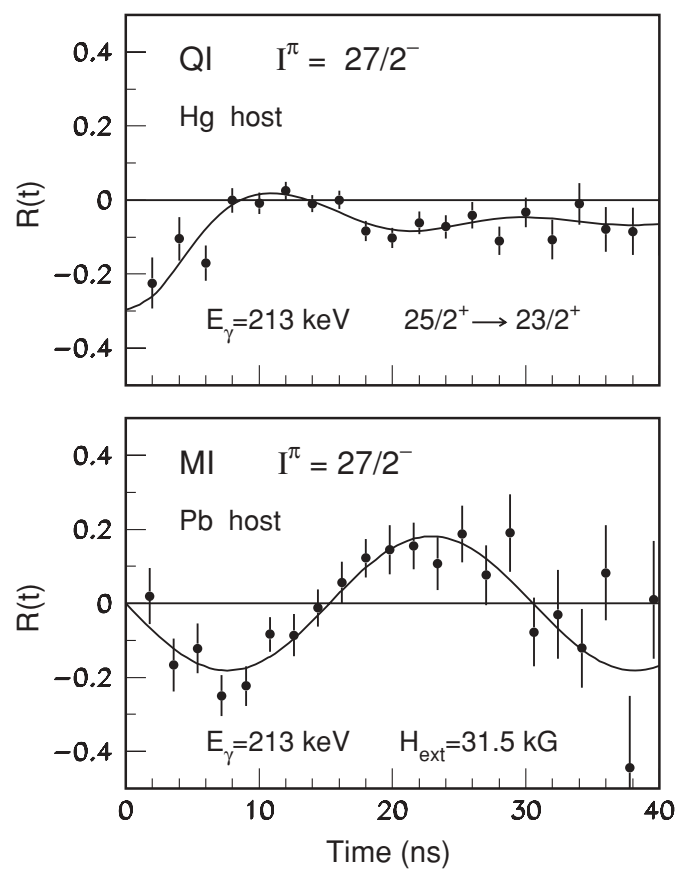

FIG. 4. Experimental and theoretical TDPAD spectra for the $27 / 2^{-} \quad T_{1 / 2}=9.4(5)$ ns isomer in ${ }^{193} \mathrm{~Pb}$ illustrating (top) the quadrupole interaction in solid $\mathrm{Hg}$ host and (bottom) the magnetic interaction in an external magnetic field. 


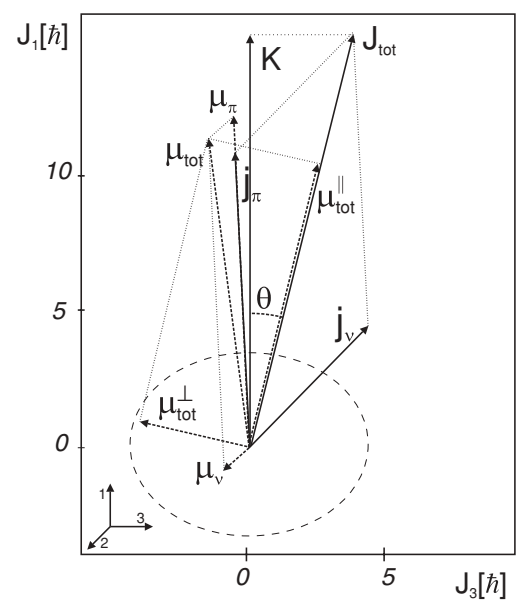

FIG. 5. Coupling scheme for the proton and neutron spins at the band head of a shears band for small oblate deformation. $j_{\pi}, j_{\nu}$, and $J_{\text {tot }}$ are the angular momenta of the proton particles, neutron holes, and the total spin; $\mu_{\pi}, \mu_{v}$, and $\mu_{\mathrm{tot}}$ are the corresponding magnetic moments; $\mu_{\mathrm{tot}}^{\|}$and $\mu_{\mathrm{tot}}^{\perp}$ are the projections of the magnetic moment along the spin axis and transverse to it; $K$ is the projection of the spin on the symmetry axis of the nucleus; and $\theta$ is the tilting angle of the spin.

an absolute value of $\left|Q_{s}\left(27 / 2^{-} ; 9.4 \mathrm{~ns}\right)\right|=2.6(3) e \mathrm{~b}$ for the spectroscopic quadrupole moment. Note that this value slightly differs from the value reported previously $[16,17]$, which is due to the fact that the spin of the isomer was reassigned. The angular distribution coefficient, $A_{2}=-0.38(9)$, which was determined for the $213-\mathrm{keV}$ transition, is in accordance with the dipole multipolarity previously assigned to it [11]. The large absolute value indicates a mixed $M 1+E 2$ multipolarity.

To provide more support to the QI analysis, which is mainly based on time spectra in the prompt region, we have also reexamined the data from the $g$-factor TDPAD measurement [10]. In that experiment the recoiling ${ }^{193} \mathrm{~Pb}$ nuclei were implanted in the cubic lattice of $\mathrm{Pb}$, placed in an external magnetic field of $31.5 \mathrm{kG}$. The Larmor precession function, $R(t)$, derived from time spectra registered at angles $\pm 135^{\circ}$ for the $213-\mathrm{keV}$ transition, is displayed in the lower panel of Fig. 4. A value of $+0.68(3)$ was derived for the $g$ factor, in perfect agreement with the previously reported value [10]. The modulation amplitude is two times larger than that found for the 158-keV E1 transition (Fig. 2 of Ref. [10]). On the basis of these results, a mixing coefficient $\delta(M 1 / E 2)=-0.13(2)$ was deduced for the $213-\mathrm{keV}$ transition. The new spin value of the $M 1$ band head, combined with the recent precise measurement of the $g$ factors of the $11^{-}$states in ${ }^{194,196} \mathrm{~Pb}$ [23], results in a remarkable agreement between the measured $g$ factor, $g_{\text {expt }}\left(27 / 2^{-} ; 9.4 \mathrm{~ns}\right)=0.68(3)$ [10], and the calculated one using the additivity approach, $g_{\text {calc }}\left(27 / 2^{-} ; 9.4 \mathrm{~ns}\right)=0.68(1)$. The coupling of the spins and corresponding magnetic moments are presented in Fig. 5.

Small quadrupole moments were recently measured for the $21 / 2^{-}$and $33 / 2^{+}$states, described by three-quasineutron configurations $[16,20]$. Similarly, the QI pattern of the $25 / 2^{-} \rightarrow$ $21 / 2^{-} 556-\mathrm{keV}$ transition shows a long quadrupole period, indicating a weak interaction strength. The short half-life of the isomer prevents precise measurement; however, an upper limit $\left|Q_{s}\left(27 / 2^{-} ; 5.3 \mathrm{~ns}\right)\right| \leqslant 0.5 e \mathrm{~b}$ was set for the quadrupole moment of this state.

\section{DISCUSSION}

The $27 / 2^{-}$shears band head in ${ }^{193} \mathrm{~Pb}$ is described by the coupling of a neutron hole in the $1 i_{13 / 2}$ orbital with the $\left(1 h_{9 / 2} 1 i_{13 / 2}\right)_{11^{-}}$proton intruder configuration, with the proton and neutron spins oriented perpendicular to each other. A notable feature of the shears states is that the excitations involved in their structure take different deformations; for example, in the $\mathrm{Pb}$ region the neutron excitations take nearspherical shapes, whereas the proton intruder excitations are moderately oblate deformed [24].

The known spectroscopic quadrupole moments in the neutron-deficient $\mathrm{Pb}$ isotopes with $A \leqslant 196$ are collected in Table I. Large quadrupole moments were measured for the $11^{-}$ isomers in ${ }^{192,194,196} \mathrm{~Pb}$ [25-28] and the $27 / 2^{-} 9.4 \mathrm{~ns}$ isomer in ${ }^{193} \mathrm{~Pb}$ (this work), as seen in Table I. These states, involving a proton intruder excitation, have quadrupole moments about an order of magnitude larger compared to those for the neutron states.

In general, the calculated $Q_{s}^{\mathrm{TAC}}$ values are in fair agreement with the experiment. The calculations were carried out within the pairing-plus-quadrupole TAC model, using the adopted parameter set for the $\mathrm{Pb}$ region, which was used to describe the yrast $M 1$ bands in the odd-mass ${ }^{193-199} \mathrm{~Pb}$ nuclei [29]. The quadrupole-quadrupole coupling constant, which controls the size of the deformation, was adjusted in each even- $A$ isotope to the experimental quadrupole moment of the corresponding near-spherical $v\left(1 i_{13 / 2}^{-2}\right)_{12^{+}}$isomer and it was interpolated for the odd-mass isotopes. With the exception of ${ }^{192} \mathrm{~Pb}$, the calculations slightly underestimate the measured moments for the $11^{-}$isomers. The $Q_{s}^{\mathrm{TAC}}$ value for the $M 1$ band head perfectly reproduces the experimental quadrupole moment.

Moderate oblate quadrupole deformations were calculated for both the $11^{-}$isomers $\left(\epsilon_{2}=-0.12\right)$ and the $27 / 2^{-}$shears state $\left(\epsilon_{2}=-0.11\right)$. The deformation of the $M 1$ band head appears, therefore, to be dominated by the polarizing effect of the two-proton particle-hole state.

The deformation parameter can also be deduced from the experimental quadrupole moment by assuming an axially symmetric deformed homogeneous charge distribution. The coupling of the spins of the protons and the neutrons is presented in Fig. 5. In such a case we may use the formulas [30]

$$
Q_{s}=Q_{0} \frac{3 K^{2}-I(I+1)}{(I+1)(2 I+1)}
$$

and

$$
Q_{0}=\frac{3}{\sqrt{5 \pi}} Z R_{0}^{2} \beta_{2}\left(1+0.36 \beta_{2}\right),
$$

where $Z$ is the proton number, $\beta_{2}$ is a deformation parameter, and the nuclear radius $R_{0}=r_{0} A^{1 / 3}$, with $r_{0}=1.2 \mathrm{fm}$. The deformation parameters $\beta_{2}$ and $\epsilon_{2}$ are related to each other as [30]

$$
\beta_{2}=\frac{4}{3} \sqrt{\frac{\pi}{5}} \epsilon_{2}\left[1+\frac{\epsilon_{2}}{3}+\left(\frac{\epsilon_{2}}{3}\right)^{2}+\cdots\right] .
$$


TABLE I. Experimental spectroscopic quadrupole moments in neutron-deficient $\mathrm{Pb}$ nuclei compared with theoretical estimates (see text). The adopted values of the isomer lifetimes [22] and absolute values for the quadrupole moments, if no sign is given, are listed.

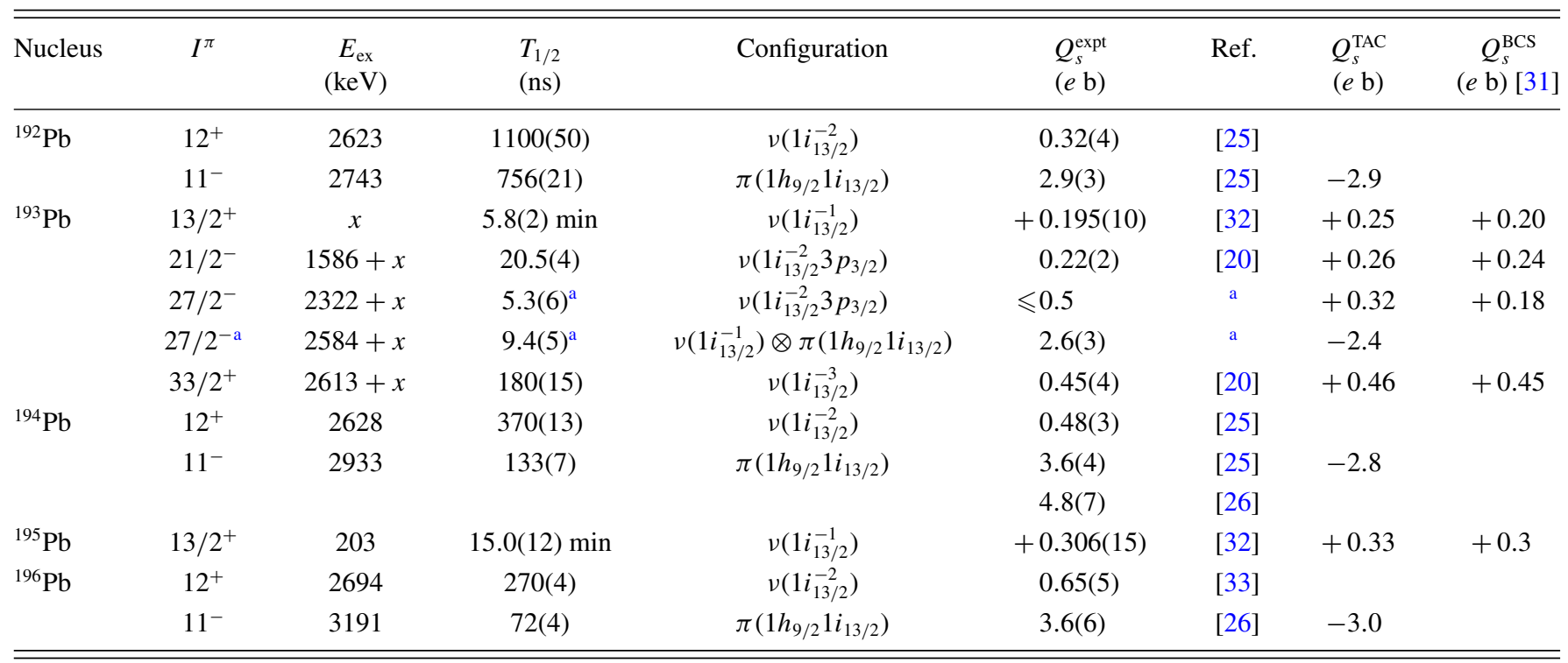

${ }^{\mathrm{a}}$ Results obtained in the present work.

The value of the calculated deformation parameter is reproduced by assuming an effective value for the $K$ quantum number, $K_{\text {eff }}=13.1(5)$, which is close to the strong-coupling limit $K=I$. From this we conclude that the tilting angle of the band is small, in agreement with the predicted value $\theta \approx 23^{\circ}$ [29].

The quadrupole moments of the neutron states were analyzed within a microscopic BCS approach in a numberprojected one- and three-quasiparticle neutron space [31]. The BCS calculations with an effective neutron charge of $e_{v}^{\text {eff }}=1.0 e$, which reproduce the quadrupole moments of neutron states in nuclei near the $N=126$ closed shell [34], underestimate the quadrupole moments in the light lead nuclei. An overall good description of the quadrupole moments in ${ }^{193} \mathrm{~Pb}$ and the neighboring light odd-mass $\mathrm{Pb}$ nuclei was obtained in Ref. [20] by using an increased effective neutron charge of $e_{v}^{\text {eff }}=2.17 e$, which reflects the increased $E 2$ polarization effect of valence neutrons when approaching the neutron midshell, as illustrated in Ref. [34]. Using the same effective neutron charge, a rather small quadrupole moment, $Q_{s}^{\mathrm{BCS}}=+0.18 e \mathrm{~b}$, is calculated for the three-quasineutron $27 / 2^{-}$state, which is consistent with the experimental upper limit.

\section{SUMMARY}

In conclusion, the pulsed-beam TDPAD method was applied for spectroscopic quadrupole moment measurements in ${ }^{193} \mathrm{~Pb}$ nuclei implanted in a $\mathrm{Hg}$ solid host. The absolute value of the quadrupole moment for the 9.4-ns isomer band head of a magnetic rotational band was determined as 2.6(3) $e \mathrm{~b}$ and an upper limit of $0.5 e \mathrm{~b}$ was set for the 5.3-ns isomer.
These are the shortest-lived isomers for which the quadrupole interaction pattern could ever be observed time differentially in in-beam experiments. The spin of the 9.4-ns isomer was fixed to $I^{\pi}=27 / 2^{-}$after angular distribution measurements. The measured quadrupole moment for the $M 1$ band head is reproduced within the pairing-plus-quadrupole TAC model. This agreement represents important new evidence that the repulsive force between the proton particle and the neutron hole orbitals, which determines the level spacing of the magnetic rotational bands, is generated by the quadrupole polarization of the core. The TAC analysis of the quadrupole moments within this model indicates that the deformation of the shears state is essentially determined by the polarization induced by the excited protons across the $Z=82$ shell gap. It will be interesting to study this effect also in other cases, for example for other configurations, or for states with a higher number of quasiparticles involved, when the tilting angle is expected to be larger.

\section{ACKNOWLEDGMENTS}

The authors thank the staff of the XTU-Tandem of Laboratori Nazionali di Legnaro for the high quality of the delivered pulsed beam. Support through the EU TMR Programme under Contract No. HPRI-CT-1999-00083; the BMBF, under Contract No. 06 BN 109; the Romanian National Authority for Scientific Research, under Contract No. IDEI52/07; the Bulgarian National Science Fund, under Grants No. DID02/16, No. DRNF02/5, and No. BRS-03/07; and the Flemish Bilateral Fund, under Contract No. BIL02/24 is acknowledged.
[1] Amita, A. K. Jain, and B. Singh, At. Data Nucl. Data Tables 74, 283 (2000).
[2] R. M. Clark and A. O. Macchiavelli, Annu. Rev. Nucl. Part. Sci. 50, 1 (2000). 
[3] H. Hübel, Prog. Part. Nucl. Phys. 54, 1 (2005).

[4] S. Frauendorf, Z. Phys. A 358, 163 (1997).

[5] S. Frauendorf, Rev. Mod. Phys. 73, 463 (2001).

[6] S. Frauendorf, Nucl. Phys. A 557, 259c (1993).

[7] G. Baldsiefen et al., Nucl. Phys. A 574, 521 (1994).

[8] A. O. Macchiavelli, R. M. Clark, P. Fallon, M. A. Deleplanque, R. M. Diamond, R. Krücken, I. Y. Lee, F. S. Stephens, A. Asztalos, and K. Vetter, Phys. Rev. C 57, 1073(R) (1998).

[9] G. Baldsiefen et al., Proceedings of the Xth International School on Nuclear Physics and Nuclear Energy, edited by W. Anderejtcheff and D. Elenkov (INRNE, Sofia, 1991), p. 10; Phys. Lett. B 275, 252 (1992).

[10] S. Chmel, F. Brandolini, R. V. Ribas, G. Baldsiefen, A. Görgen, M. De Poli, P. Pavan, and H. Hübel, Phys. Rev. Lett. 79, 2002 (1997).

[11] J. M. Lagrange, M. Pautrat, J. S. Dionisio, Ch. Vieu, and J. Vanhorenbeeck, Nucl. Phys. A 530, 437 (1991).

[12] G. Baldsiefen et al., Phys. Rev. C 54, 1106 (1996).

[13] L. Ducroux et al., Z. Phys. A 356, 241 (1996).

[14] N. Fotiades et al., Phys. Rev. C 57, 1624 (1998).

[15] G. D. Dracoulis, G. J. Lane, T. Kibédi, and P. Nieminen, Phys. Rev. C 79, 031302(R) (2009).

[16] D. L. Balabanski et al., Eur. Phys. J. A 20, 191 (2004).

[17] D. L. Balabanski, AIP Conf. Proc. 831, 268 (2006).

[18] R. Vianden, Hyperfine Interact. 35, 1079 (1987).
[19] H.-E. Mahnke et al., Phys. Lett. B 88, 48 (1979).

[20] M. Ionescu-Bujor et al., Phys. Rev. C 70, 034305 (2004).

[21] E. Dafni, R. Bienstock, M. H. Rafailovich, and G. D. Sprouse, At. Data Nucl. Data Tables 23, 315 (1979).

[22] Data available at [http://www.nndc.bnl.gov/ensdf/].

[23] K. Vyvey et al., Phys. Rev. C 69, 064318 (2004).

[24] N. Smirnova, P. H. Heenen, and G. Neyens, Phys. Lett. B 569, 151 (2003).

[25] M. Ionescu-Bujor et al., Phys. Lett. B 650, 141 (2007).

[26] K. Vyvey, D. L. Balabanski, and G. Neyens, Eur. Phys. J. A 22, 501 (2004).

[27] K. Vyvey, D. Borremans, N. Coulier, R. Coussement, G. Georgiev, S. Teughels, G. Neyens, H. Hübel, and D. L. Balabanski, Phys. Rev. C 65, 024320 (2002).

[28] K. Vyvey et al., Phys. Rev. Lett. 88, 102502 (2002).

[29] S. Chmel, S. Frauendorf, and H. Hübel, Phys. Rev. C 75, 044309 (2007).

[30] K. E. G. Löbner, M. Vetter, and V. Hönig, Nucl. Data Tables A 7, 495 (1970).

[31] C. A. P. Ceneviva, L. Losano, N. Teruya, and H. Dias, Nucl. Phys. A 619, 129 (1997).

[32] S. B. Dutta et al., Z. Phys. A 341, 39 (1991).

[33] S. Zywietz, H. Grawe, H. Haas, and M. Menningen, Hyperfine Interact. 9, 109 (1981).

[34] G. Neyens, Rep. Prog. Phys. 66, 633 (2003). 